\title{
Ultrasonic evaluation of muscle functional recovery following free functioning gracilis transfer, a preliminary study
}

Yi Hou', Jiantao Yang ${ }^{2}$, Bengang Qin², Liqiang Gu ${ }^{2^{*}}$ and Jia Zheng ${ }^{1^{*}}$

\begin{abstract}
Background: Ultrasonic measurement has not been utilized to assess the functional recovery of transplanted muscle. This study aimed to investigate the feasibility of using B-ultrasound measurement to assess muscle recovery following free functioning gracilis transfer.

Methods: From January 2009 to January 2014, 35 patients receiving free functioning gracilis transfer to treat total brachial plexus injury were enrolled. B-ultrasound was adopted to determine the cross-sectional area (CSA) of transplanted gracilis muscle at rest and contraction state. The ratio of pre- to post-transplant CSA value at rest state was defined as muscle bulk ratio (MBR). The ratio of CSA value at contraction state to rest state was defined as contraction ratio (CR).

Results: Patients with muscle strength $M \geq 4$ had significantly higher CR1 (post-transplant), CR2 (pre-transplant), and range of motion (ROM, joint mobility) than those with muscle strength $M<4$. The CR1 $>$ CR2 group had significantly higher CR1, muscle strength, and ROM than the CR1 $\leq$ CR2 group. The MBR $>1$ group had significantly higher muscle strength than the $M B R \leq 1$ group. CR1 value was highly correlated with muscle strength and with ROM. CR2 value was moderately correlated with muscle strength and ROM. Multivariate linear regression analysis showed that a higher CR1/CR2 value was associated with a higher muscle strength and joint mobility. The CR1 > CR2 group had better muscle strength and ROM than the CR1 $\leq$ CR2 groups.
\end{abstract}

Conclusion: B-ultrasound measurement can quantitatively reflect muscle strength following gracilis transfer, and CR value could be a potential indicator for functional recovery of the transplanted gracilis muscle.

Level of Evidence: Prognostic studies, Level II.

Keywords: Ultrasound, Muscle contraction, Functional muscle transplantation, Muscle strength

\section{Background}

Free functioning gracilis transfer is a surgical procedure for limb motor function in patients with Brachial plexus avulsion, brachial plexus nerve injuries, traumatic muscle

\footnotetext{
*Correspondence: guliqiang1963@aliyun.com; zhengjia90180@sina.com ${ }^{1}$ Department of Orthopedics, Henan Provincial People's Hospital, No. 7 , Weiwu Road, Zhengzhou 450003, China

${ }^{2}$ Department of Microsurgery and Orthopedic Trauma, the First Affiliated Hospital of Sun Yat-Sen University, 58 Second Zhongshan Road, Guangzhou 510080, China
}

loss, facial paralysis, and tumor resection [1-8]. It transplants gracilis muscles with vascular nerve pedicles to the upper limbs, suturing the affected blood vessels and motor nerves to replace the function of lost muscles in the affected area [9]. The reinnervation after gracilis muscle transfer is a slow process. Based on the recovery condition, auxiliary surgery (such as fusion of wrist joint) is usually required to promote functional recovery $[10,11]$. Therefore, timely assessing functional recovery of transplanted muscle can provide a reference for subsequent decision-making. 
Currently, assessment methods for functional recovery of transplanted muscle include electromyography [12], manual muscle test (MMT) [13], and magnetic resonance imaging (MRI) [14]. However, all these evaluation methods have some defects. EMG results are not always consistent with muscle strength recovery of the transplanted gracilis muscle. MMT is easy to conduct but subjective, and muscle strength M4 cannot be quantitatively assessed. MRI cannot dynamically assess muscle contraction function, and it is expensive.

B-ultrasound is non-invasive, convenient, and cheap, and can reflect muscle strength by echo density, muscle thickness, cross-sectional area, and intermuscular volume [15-19]. Compared to the static observation of MRI, B-ultrasound can observe the dynamic contraction of muscle. Ultrasonic measurement can easily obtain morphological parameters of muscle, such as muscle thickness and cross-sectional area. The cross-sectional area measured by B-ultrasound has been used to investigate the effect of resistance training on muscle strength $[16$, 20]. Since muscle morphological parameters are closely related to muscle function, muscle strength and function can be reflected by morphological parameters in ultrasonic measurement [20]. The correlation between ultrasonic morphologic parameters and muscle strength has been demonstrated [16, 21, 22]. However, B-ultrasound has not been used to determine functional recovery of transplanted muscle following. In this study, therefore, we aimed to investigate the feasibility of B-ultrasound measurement for evaluation of muscle recovery following free functioning gracilis transfer.

\section{Methods}

\section{Participants}

From January 2009 to January 2014, 35 patients receiving free functioning gracilis transfer to treat total brachial plexus injury in our hospital were enrolled. All patients received free functioning gracilis transfer for the first time. This study was approved by the institutional review board of our hospital. Written informed consent was obtained from each patient.

\section{Surgical procedure}

All patients underwent free functioning gracilis transfer to reconstruct the functions of elbow flexion-finger/ thumb extension, and the ipsilateral accessory nerve was used as a donor motor nerve. The reconstruction of the transplanted gracilis muscle was located from the lateral clavicle to the extensor carpi radialis longus muscle and the tendon of extensor pollicis longus muscle at the dorsal side of the distal forearm. The gracilis muscle was completely harvested from the origin on the pubic ramus to the pes anserine tendon around the interior knee joint, which length can meet the needs of reconstruction (Additional file 1: Figure S1). Patient's elbow was flexed in $90^{\circ}$, and the gracilis muscle was placed on the shoulder-upper arm-anterolateral elbow-the dorsal side of the middle $1 / 3$ of the forearm. The proximal end of the gracilis muscle was fixed to the acromion or outer clavicle periosteum, spanning the elbow joint, and the distal end was sutured to extensor digitorum communis and extensor pollicis longus tendons with appropriate tension. The elbow joint was maintained at functional position by plaster cast. Therefore, the gracilis muscle can be used for long-distance reconstruction without tendon grafts, and the functions of elbow flexion, finger extension, and thumb extension can be simultaneously reconstructed (Additional file 2: Figure S2). If necessary, the other gracilis muscle can be placed in the forearm to reconstruct the function of extrinsic muscles of the hand. The purpose of double gracilis transplantation was to restore the grasping function of the hand to a greater extent.

\section{Muscle strength measurement and range of motion (ROM)}

The muscle strength was assessed by manual muscle strength test and the ROM of elbow flexion was determined by a protractor tool. All assessment was performed by a trained physician who was not involved in the ultrasonic measurement. To avoid observation bias, the definition of M4 strength was modified to "muscle can resist at least the examiner's one finger or at least $1 \mathrm{~kg}$ in weight" according to Lin et al's study [23].

\section{Ultrasonic measurement and outcome analysis}

All B-ultrasound examinations are performed using the Venue 40 Ultrasound (GE Healthcare, USA) and the cross-sectional area (CSA) of the with gracilis muscle was obtained based on the ultrasound images with the Scion Image software (National Institutes of Health, Bethesda $\mathrm{MD})$. All the process was performed by the same physician and can be completed within $10 \mathrm{~min}$. Before and after transplantation, the ultrasound examinations and the corresponding CSA of transplanted gracilis muscle were measured at rest state and contraction state. Each ultrasound examination was continuously repeated five times and the corresponding CSA value was averaged.

The origin and midpoint of the abdominal muscle were determined with B-ultrasound. Before transplantation, the CSA was measured at the midpoint of the abdominal muscle. Before transplantation, the CSA of the gracilis muscle at rest state was measured at the neutral position of the hip joint, while the CSA of the gracilis muscle at the contraction state was determined at $30^{\circ}$ abduction of the hip joint.

For CSA measurement after transplantation, the patient was placed in the supine position, and the 
upper extremity was straightened. The midpoint of the transplanted gracilis muscle was determined by B-ultrasound scan, and the plane was used to measure the CSA of the gracilis muscle at rest state. The CSA of the gracilis muscle at the contraction state was determined in the isometric contraction state with shoulder abduction of $20^{\circ}$ and elbow flexion at $60^{\circ}$, which was the posture used to restore the original length of the transplanted muscle during surgery. Restoring the original length of the transplanted muscle in this position will not cause the elbow joint straightening obstacle, and can achieve the best elbow flexion-finger extension effect. Therefore, patients received B-ultrasound examination in the same posture to make the pre-transplant and post-transplant results comparable.

\section{Muscle bulk ratio (MBR) and contraction ratio (CR)}

The ratio of pre- to post-transplant CSA value of gracilis muscle at rest state was defined as MBR. The ratio of contraction state to rest state CSA value was defined as contraction ratio (CR). The CR values of gracilis muscle before and after transplantation were defined as CR-2 and CR-1, respectively (Fig. 1). The patients were divided into following dichotomous groups for subgroup analysis of muscle strength or ROM: the MBR $>1$ vs. $M B R<1$; CR-1 $>$ CR-2 group vs. CR-1 $<$ CR-2 group; and muscle strength $\geq M 4$ vs. muscle strength $<$ M 4 groups.
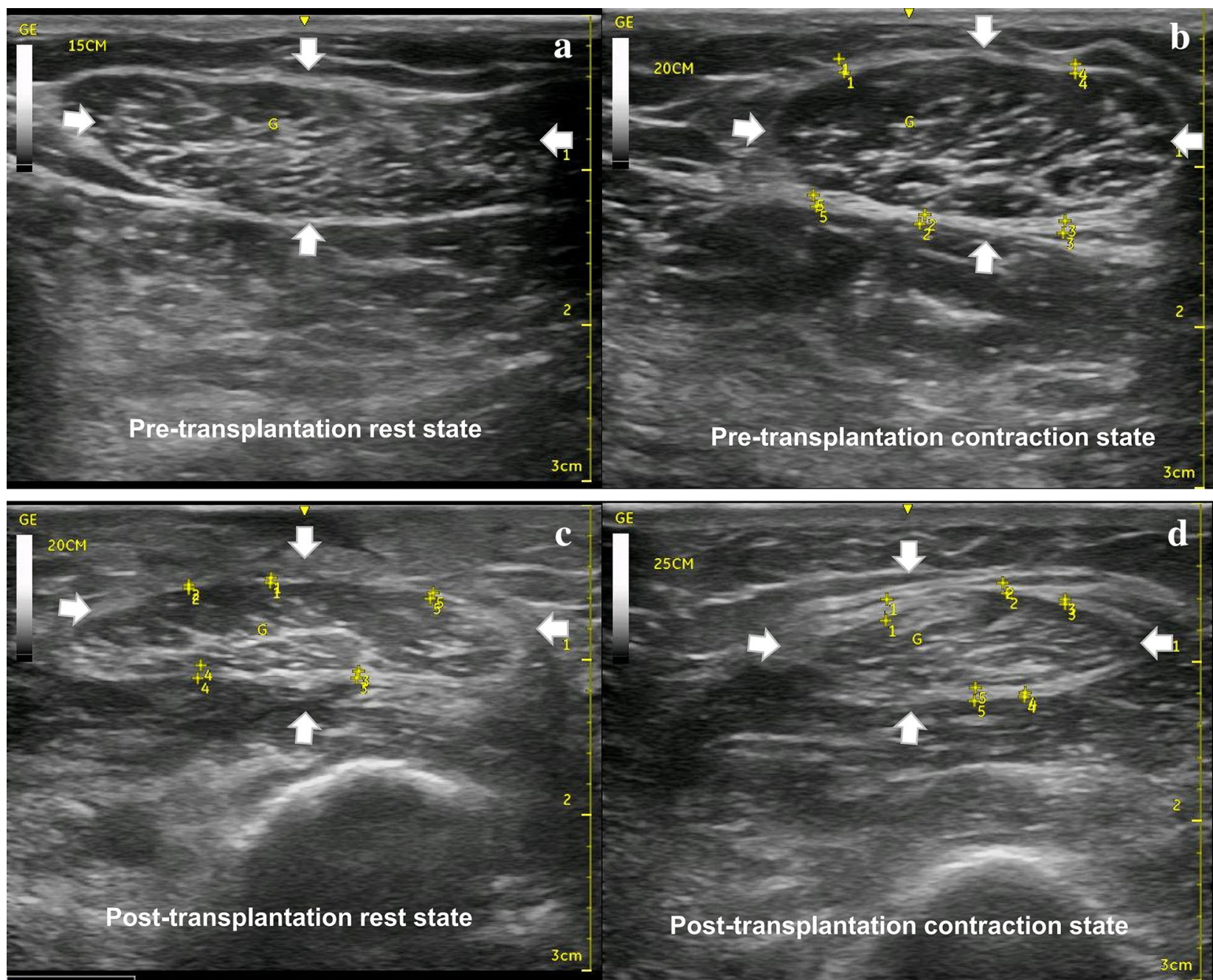

Fig. 1 Ultrasound image of cross-sectional area of the gracilis muscle. The white arrows indicate muscle cross-section area (CSA). a Pre-transplantation rest state; $\mathbf{b}$ Pre-transplantation contraction state; $\mathbf{c}$ post-transplantation rest state; $\mathbf{d}$ post-transplantation contraction state. The ratio of pre- to post-transplant CSA value of gracilis muscle at rest state was defined as muscle bulk ratio (MBR). The ratio of CSA value at the contraction state to that at rest state was defined as contraction ratio (CR). The CR values of gracilis muscle before and after transplantation were defined as $C R-2$ and $C R-1$, respectively. $M B R=C S A$ in $\mathbf{c} / C S A$ in $\mathbf{a}$; $C R 1=C S A$ in $\mathbf{d} / C S A$ in $\mathbf{c}$; $C R 2=C S A$ in $\mathbf{b} / C S A$ in $\mathbf{a}$ 


\section{Statistical analysis}

Continuous variables are presented as mean \pm standard deviation (SD), while categorical data are shown as number and percentage (\%). Student's independent t-test was used to compare the differences between groups. If normality of continuous variables was not assumed, non-parametric analysis Mann-Whitney $U$ test would be used instead. Chi-square test and Fisher's exact test (if any expected value lower than 5 was observed) were used for categorical data. Pearson's correlation coefficient was used to report the correlation among continuous variables. Univariate and multivariate linear regression models were used to investigate the associations between $\mathrm{CR} / \mathrm{MBR}$ and muscle strength/range of motion (ROM). A $P$-value lower than 0.05 would be recognized as significance. All analyses were performed using IBM SPSS Version 20 (SPSS Statistics V20, IBM Corporation, Somers, New York).

\section{Results}

\section{Patient's clinical characteristics}

A total of 35 patients (32 males and 3 females, mean age $=30.40 \pm 8.40$ years) undergoing free functioning gracilis transfer were included. The mean follow-up period of all patient's was $19.11 \pm 6.51$ (range 8 to 32 ) months after transplantation. Table 1 demonstrates demographic

Table 1 Patient's clinical characteristics

\begin{tabular}{ll}
\hline Parameters & Mean \pm SD / N (\%) \\
\hline Gender & \\
Male & $32(91.43)$ \\
Female & $3(8.57)$ \\
Age, years & $30.40 \pm 8.40$ \\
Follow-up period, month & $19.11 \pm 6.51$ \\
Muscle strength (M) & $3.26 \pm 0.89$ \\
Muscle strength group & \\
M<4 & $17(48.57)$ \\
M $\leq 4$ & $18(51.43)$ \\
CR1 & $1.23 \pm 0.15$ \\
CR2 & $1.22 \pm 0.13$ \\
Relation between CR1 and CR2 & \\
CR1 >CR2 & $20(57.14)$ \\
CR1 $\leq$ CR2 & $15(42.86)$ \\
MBR & $1.00 \pm 0.14$ \\
MBR group & \\
$>1$ & $18(51.43)$ \\
$\leq 1$ & $17(48.57)$ \\
ROM & $73.66 \pm 27.39$ \\
\hline CR &
\end{tabular}

$C R$ contraction ratio, $M B R$ muscle bulk ratio, $R O M$ range of motion $C R 1$ was the post-transplant $C R$ value, while $C R 2$ was the pre- transplant $C R$ value and clinical characteristics, including muscle strength (M), CR1 (post-transplant), CR2 (pre-transplant), MBR, and ROM. The detailed ultrasonic measurement outcomes and muscle strength of all 35 patients are summarized in Additional file 3: Table S1.

\section{Subgroup analysis stratified by muscle strength, relative $\mathrm{CR}$, and MBR}

The ultrasonic measurement outcomes and muscle strength were compared by the following dichotomous groups, including muscle strength $(M<4$ vs. $M \geq 4)$, $\mathrm{CR}$ value order $(\mathrm{CR} 1>\mathrm{CR} 2$ vs. $\mathrm{CR} 1 \leq \mathrm{CR} 2)$, and $\mathrm{MBR}$ $(M B R>1$ vs. $M B R \leq 1)$. It was found that the $M \geq 4$ group had significantly higher CR1, CR2, and ROM values than the $\mathrm{M}<4$ group (all $P<0.001$, Table 2 ). The CR1 $>$ CR2 group had significantly higher CR1, muscle strength, and ROM than the CR1 $\leq$ CR2 group (all $P<0.01$, Table 3 ). The MBR $>1$ group had significantly higher muscle strength than the $M B R \leq 1$ group $(P=0.038$, Table 4$)$.

\section{Associations between CR/MBR and M/ROM}

To further evaluate the potential of ultrasonic outcomes as the indicator for muscle strength, correlation analysis between CR/MBR and muscle strength/ROM was performed. As shown in Table 5, CR1 had the highest correlation coefficient with muscle strength (0.808) and ROM (0.847) (both $P<0.01$ ); CR2 had a medium

Table 2 Comparisons between muscle strength groups

\begin{tabular}{lccc}
\hline Parameters & $\boldsymbol{M}<\mathbf{4}$ & $\boldsymbol{M} \geq \mathbf{4}$ & $\boldsymbol{P}$ \\
\hline CR1 & $1.10 \pm 0.06$ & $1.35 \pm 0.10$ & $<0.001$ \\
Muscle strength & $2.47 \pm 0.62$ & $4.00 \pm 0.00$ & $<0.001$ \\
ROM & $52.12 \pm 22.22$ & $94.00 \pm 11.54$ & $<0.001$ \\
CR2 & $1.15 \pm 0.09$ & $1.30 \pm 0.12$ & $<0.001$ \\
MBR & $1.02 \pm 0.18$ & $0.99 \pm 0.09$ & 0.576 \\
\hline
\end{tabular}

$C R$ contraction ratio, $M B R$ muscle bulk ratio, $R O M$ range of motion

$C R 1$ was the post-transplant $C R$ value, while $C R 2$ was the pre-transplant $C R$ value

Table 3 Comparisons between comparative relations of CR groups

\begin{tabular}{lccl}
\hline Parameters & CR1 $>$ CR2 & CR1 $\leq$ CR2 & $P$ \\
\hline CR1 & $1.29 \pm 0.15$ & $1.14 \pm 0.11$ & 0.002 \\
Muscle strength & $3.60 \pm 0.68$ & $2.80 \pm 0.94$ & 0.006 \\
ROM & $85.20 \pm 22.40$ & $58.27 \pm 26.39$ & 0.003 \\
CR2 & $1.21 \pm 0.12$ & $1.24 \pm 0.15$ & 0.483 \\
MBR & $0.98 \pm 0.11$ & $1.04 \pm 0.17$ & 0.237 \\
\hline
\end{tabular}

$C R$ contraction ratio, $M B R$ muscle bulk ratio, $R O M$ range of motion $C R 1$ was the post-transplant $C R$ value, while $C R 2$ was the pre-transplant $C R$ value 
Table 4 Comparisons between MBR groups

\begin{tabular}{lccr}
\hline Parameters & MBR $>\mathbf{1}$ & MBR $\leq \mathbf{1}$ & \multicolumn{1}{c}{} \\
\hline CR1 & $1.24 \pm 0.14$ & $1.21 \pm 0.17$ & 0.660 \\
Muscle strength & $3.56 \pm 0.51$ & $2.94 \pm 1.09$ & 0.038 \\
ROM & $78.67 \pm 16.98$ & $68.35 \pm 35.06$ & 0.272 \\
CR2 & $1.24 \pm 0.11$ & $1.21 \pm 0.15$ & 0.500 \\
MBR & $1.11 \pm 0.11$ & $0.89 \pm 0.05$ & $<0.001$ \\
\hline
\end{tabular}

$C R$ contraction ratio, $M B R$ muscle bulk ratio, $R O M$ range of motion

CR1 was the post-transplant $C R$ value, while $C R 2$ was the pre- transplant $C R$ value

Table 5 Correlation coefficient results

\begin{tabular}{llll}
\hline Parameters & CR1 & CR2 & MBR \\
\hline Muscle strength & $0.808^{* *}$ & $0.491^{* *}$ & 0.204 \\
ROM & $0.847^{* *}$ & $0.556^{* *}$ & 0.017 \\
\hline
\end{tabular}

$C R$ contraction ratio, $M B R$ muscle bulk ratio; $R O M$ range of motion

CR1 was the post-transplant CR value, while CR2 was the pre- transplant CR value

**P $<0.001$

correlation coefficient with $\mathrm{M}(0.491)$ and ROM (0.556) (both $P<0.01)$. However, MBR had no significant correlation with muscle strength and ROM (both $P>0.05$ ). The coefficient between $\mathrm{M}$ and ROM was $0.903(P<0.001)$.

The associations between CR/MBR and M/ROM were further evaluated by univariate and multivariate linear regression analyses. Multivariate linear regression analysis adjusted for patient's age and gender showed that a higher CR1/CR2 value suggested a higher muscle strength and ROM value (all $P<0.01$, Table 6). Meanwhile, the CR1 $>$ CR2 group had a better muscle strength and ROM than the CR1 $\leq$ CR2 groups (all $P<0.01$, Table 6). However, there were no significant results in the regression models of MBR (all $P>0.05$ ).

\section{Discussion}

In this study, we investigated the feasibility of B-ultrasound to evaluate the muscle recovery following free functioning gracilis transfer. The results showed that The $M \geq 4$ group had significantly higher CR1, CR2, and ROM value (joint mobility) as compared with the $M<4$ group. The CR1 > CR2 group had significantly higher CR1, muscle strength, and ROM than the CR1 $\leq$ CR2 group. The $M B R>1$ group had significantly higher muscle strength than the $\mathrm{MBR} \leq 1$ group. CR1 value was highly correlated with muscle strength $(r=0.808)$ and ROM $(r=0.847)$, while CR2 value was moderately correlated with muscle strength $(r=0.491)$ and ROM $(r=0.556)$. Multivariate linear regression analysis showed that a higher CR1/ CR2 value was associated with a higher muscle strength and higher joint mobility. Meanwhile, the CR1 $>$ CR2 group had better muscle strength and ROM than the $\mathrm{CR} 1 \leq \mathrm{CR} 2$ groups (all $P<0.01$ ). Taken together, these results suggested that $\mathrm{B}$-ultrasound measurement can quantitatively reflect muscle function following gracilis

Table 6 Univariate and multivariate linear regression results

\begin{tabular}{|c|c|c|c|c|}
\hline \multirow[b]{2}{*}{ Parameters } & \multicolumn{2}{|l|}{ Univariate } & \multicolumn{2}{|l|}{ Multivariate $^{1}$} \\
\hline & $B^{2}$ & $P$ & $B$ & $P$ \\
\hline \multicolumn{5}{|c|}{ Muscle strength } \\
\hline CR1 & $4.70(3.49-5.91)$ & $<0.001$ & $4.61(3.37-5.84)$ & $<0.001$ \\
\hline CR2 & $3.35(1.24-5.45)$ & 0.003 & $3.47(1.35-5.59)$ & 0.002 \\
\hline \multicolumn{5}{|c|}{ Relation between CR1 and CR2 } \\
\hline$C R 1>C R 2$ & ref & - & ref & - \\
\hline $\mathrm{CR} 1 \leq \mathrm{CR} 2$ & $-0.80(-1.36$ to 0.24$)$ & 0.006 & $-0.80(-1.39$ to 0.21$)$ & 0.010 \\
\hline MBR & $1.30(-0.90$ to 3.51$)$ & 0.239 & $1.50(-0.75-3.74)$ & 0.183 \\
\hline \multicolumn{5}{|l|}{ ROM } \\
\hline CR1 & $152.31(118.49-186.13)$ & $<0.001$ & $150.44(115.59-185.29)$ & $<0.001$ \\
\hline CR2 & $117.16(55.06-179.27)$ & $<0.001$ & $122.02(59.24-184.79)$ & $<0.001$ \\
\hline \multicolumn{5}{|c|}{ Relation between CR1 and CR2 } \\
\hline$C R 1>C R 2$ & ref & - & ref & - \\
\hline $\mathrm{CR} 1 \leq \mathrm{CR} 2$ & $-26.93(-43.73$ to 10.14$)$ & 0.003 & $-27.34(-45.41$ to 9.27$)$ & 0.004 \\
\hline MBR & $3.26(-66.37$ to 72.90$)$ & 0.925 & $7.87(-64.01$ to 79.74$)$ & 0.825 \\
\hline
\end{tabular}

$C R$ contraction ratio, $M B R$ muscle bulk ratio, $R O M$ range of motion

$C R 1$ was the post-transplant $C R$ value, while $C R 2$ was the pre-transplant $C R$ value

1 Multivariate results were adjusted with patient's age and gender

2 B stands for regression coefficient 
transfer, and CR could be a potential indicator for muscle function recovery.

Currently, ultrasonic parameters which could be used as the indicator for muscle contraction include echo intensity (EI) [21], muscle thickness [24], muscle fiber pennation [25], and measures of muscle architecture [19]. EI reflects muscle function by the density of high echo signals in the muscle but is susceptible to be affected by the connective tissue between the subcutaneous and muscle bundles. A study on the changes in muscle strength by Jacobs et al. has confirmed that EI is not an optimal indicator of muscle strength [21]. In addition, the transplanted gracilis muscle undergoes denervation and fibrosis; therefore, EI is not suitable for function evaluation following free functioning gracilis transfer. Muscle fiber pennation reflects the muscle contraction by the angle between the direction of muscle fibers and the long axis of the muscle.[26] Gracilis muscle is a non-feathery muscle, and could not be measured by muscle fiber pennation. The transplanted muscle is close to the skins, and the ultrasonic test is susceptible to be affected by probeinduced pressure; hence, muscle thickness measurement is also not applicable for muscle transplantation.

The gracilis muscle is non-feathery muscle, and its muscle fibers are arranged in the same direction as the tendon. During the muscle contraction, all the muscle fibers slide in parallel. Therefore, the histological crosssectional area of the muscle fibers of the gracilis muscle is substantially the same as the gross anatomical crosssectional area [19]. Therefore, B-ultrasound can be used to measure CSA, and the dynamic contraction of muscles can be reflected by CSA changes (i.e., CR) at rest and contraction. The transplanted gracilis muscle undergoes denervation and nerve re-innervation, and the muscle volume changes during this process. The MBR and CR are standardized indicators calculated based on CSA, which can eliminate the impact of muscle volume change during denervation and nerve re-innervation [27]. Hence, we chose CR and MBR as the indicators to explore the recovery of gracilis muscle functional after free functioning gracilis transfer.

Our results showed a high correlation between CR value and muscle function indexes, indicating that CR can be used to dynamically evaluate the recovery of muscle function after transplantation. In addition, muscle strength and ROM were significantly higher in the $C R 1>C R 2$ group than in the $C R 1 \leq C R 2$ group, suggesting that patient with elevated muscle $C R$ after transplantation has a better recovery of muscle strength and joint mobility. We observed an increase in the CR value of the gracilis muscle after transplantation. One of the possible reasons is as follows: since the motion range of elbow bowing is larger than hip adduction, the muscle fibers of transplanted gracilis muscle need to be parallel sliding for a longer distance in the elbow bowing. Therefore, the CR value was elevated after transplantation. Our results showed that the mean CR1 values of the muscle strength $\geq M 4$ group and the CR1 > CR2 group were $1.35 \pm 0.10$ and $1.29 \pm 0.15$, respectively. Based on these results, we propose that CR1 of 1.3 might be an important reference value for transplanted muscle recovery. When the $C R$ value of transplanted muscle reaches 1.3 , the muscle might have satisfactory recovery. However, this reference value should be further validated in a large trial.

In this study, we also evaluated MBR, which reflects muscle volume change, i.e., atrophy $(\mathrm{MBR}<1)$ or hypertrophy $(M B R>1)$. We found that patients who had no atrophy with gracilis muscle $(M B R>1)$ showed no significant advantage in muscle strength and joint mobility. Subgroup analysis of muscle strength also showed that MBR value was not significantly different between two muscle strength groups. Correlation analysis also revealed that there was no correlation between MBR and muscle strength/joint mobility. All these results suggested that MBR cannot be used as an indicator for muscle recovery following free functioning gracilis transfer.

Our preliminary findings demonstrated the feasibility of B-ultrasound for assessing functional recovery after gracilis transfer. Early muscle contraction changes may not be easily detected by physical examination. B-ultrasound examination can dynamically detect the progress of muscle contraction recovery, as well as the tendongliding function. When a lower postoperative $C R$ value is found in early postoperative period, the patients can receive physical therapy to promote nerve reinnervation. In the late postoperative period (e.g., at 1 year after surgery, there is no progress in muscle strength for 3 consecutive months), a second femoral muscle transplantation could be performed to rebuild the flexor. Even though this was a prospective study, the sample size was relatively small. In the future, a large trial should be conducted to validate the findings of this study.

\section{Conclusions}

In summary, our findings demonstrated that B-ultrasound measurement can quantitatively reflect muscle function following gracilis transfer, and CR value could be a potential indicator for muscle function recovery. The $\mathrm{CR}$ value of transplanted muscle was highly correlated with the muscle function, which can be used to dynamically assess muscle recovery after muscle transplantation. Elevated CR value of gracilis muscle after transplantation suggested a better prognosis. 


\section{Supplementary information}

The online version contains supplementary material available at https://doi. org/10.1186/s40001-020-00473-8.

Additional file 1: Figure S1. Design of functional free gracilis muscle transfer. The gracilis muscle iscompletely harvested from the origin on the pubic ramus to the pes anserine tendon around theinterior knee joint.

Additional file 2: Figure S2. (A) Elbow flexion and fingers and thumb extension reconstruction; (B). Fingers and thumb reconstruction.

Additional file 3: Table S1. Ultrasound and follow-up results of all 35 patients

\section{Abbreviations}

MMT: Manual muscle test; MRI: Magnetic resonance imaging; ROM: Range of motion; CSA: Cross-sectional area; MBR: Muscle bulk ratio; CR: Contraction ratio.

\section{Acknowledgements}

None declared.

\section{Authors' contributions}

The authors declare that they all have participated actively in this study and they meet the requirements of the authorship. LG and JZ designed the study and wrote the protocol. YH and JY acquired and interpreted the data. BQ managed the literature searches and analyses. YH wrote the first draft of the manuscript. All authors read and approved the final manuscript.

\section{Funding}

The current study is supported by the National Science Foundation of China NSFC (No. 81871787).

\section{Availability of data and materials}

All the data and materials have been presented in the main paper.

\section{Ethics approval and consent to participate}

This study was approved by the institutional review board of the First Affiliated Hospital of Sun Yat-Sen University (No. [2014]161]). Written informed consent was obtained from each patient.

\section{Consent for publication}

Written informed consent was obtained from each patient for the publication of this study.

\section{Competing interests}

The authors declare that they have no competing interests.Additional file 1: Figure S1. Design of functional free gracilis muscle transfer. The gracilis muscle is completely harvested from the origin on the pubic ramus to the pes anserine tendon around the interior knee joint.

Received: 22 September 2020 Accepted: 15 December 2020

Published online: 05 February 2021

\section{References}

1. Soldado F, Bertelli J. Free gracilis transfer reinnervated by the nerve to the supinator for the reconstruction of finger and thumb extension in longstanding C7-T1 brachial plexus root avulsion. J Hand Surg Am. 2013:38:941-6.

2. Organek AJ, Klebuc MJ, Zuker RM. Indications and outcomes of free tissue transfer to the lower extremity in children: Review. J Reconstr Microsurg. 2006;22:173-81

3. Hou Y, Qin B, Yang J, Li X, Yang Y, Fu G, et al. Restoration of finger and thumb movement using one-stage free muscle transplantation. J Plast Surg Hand Surg. 2016:50:130-4.
4. El-Gammal TA, El-Sayed A, Kotb MM, Saleh WR, Ragheb YF, Refai O, et al. Free functioning gracilis transplantation for reconstruction of elbow and hand functions in late obstetric brachial plexus palsy. Microsurgery. 2015;35:350-5.

5. Estrella EP, Montales TD. Functioning free muscle transfer for the restoration of elbow flexion in brachial plexus injury patients. Injury. 2016:47:2525-33.

6. Maldonado AA, Kircher MF, Spinner RJ, Bishop AT, Shin AY. Free functioning gracilis muscle transfer with and without simultaneous intercostal nerve transfer to musculocutaneous nerve for restoration of elbow flexion after traumatic adult brachial Pan-Plexus injury. J Hand Surg Am. 2017:42:293.

7. Nicoson MC, Franco MJ, Tung TH. Donor nerve sources in free functional gracilis muscle transfer for elbow flexion in adult brachial plexus injury. Microsurgery. 2017;37:377-82.

8. Takushima A, Harii K, Asato H, Ueda K, Yamada A. Neurovascular free-muscle transfer for the treatment of established facial paralysis following ablative surgery in the parotid region. Plast Reconstr Surg. 2004;113:1563-72.

9. Barrie KA, Steinmann SP, Shin AY, Spinner RJ, Bishop AT. Gracilis free muscle transfer for restoration of function after complete brachial plexus avulsion. Neurosurg Focus. 2004;16:E8.

10. Coulet B, Boch C, Boretto J, Lazerges C, Chammas M. Free Gracilis muscle transfer to restore elbow flexion in brachial plexus injuries. Orthop Traumatol Surg Res. Elsevier Masson; 2011:97:785-92. https://www.sciencedir ect.com/science/article/pii/S1877056811002301. Accessed 22 Apr 2019

11. Elzinga K, Zuo KJ, Olson JL, Morhart M, Babicki S, Chan KM. Double free gracilis muscle transfer after complete brachial plexus injury: first Canadian experience. Plast Surg (Oakville, Ont). 2014;22:26-9.

12. Scaglioni MF, Verdini F, Marchesini A, Neuendorf AD, Coccia D, Leo T, et al. Assessment of functional outcomes of temporalis muscle transfers for patients with longstanding facial paralysis. Head Neck. 2016;38:E1535-43.

13. Davidge KM, Yee A, Moore AM, Mackinnon SE. The supercharge end-to-side anterior interosseous-to-ulnar motor nerve transfer for restoring intrinsic function: clinical experience. Plast Reconstr Surg. 2015:136:344e-52e.

14. Kozin SH, Chafetz RS, Shaffer A, Soldado F, Filipone L. Magnetic resonance imaging and clinical findings before and after tendon transfers about the shoulder in children with residual brachial plexus birth palsy: a 3-year follow-up study. J Pediatr Orthop. 2010;30:154-60.

15. Henderson REA, Walker BF, Young KJ. The accuracy of diagnostic ultrasound imaging for musculoskeletal soft tissue pathology of the extremities: a comprehensive review of the literature. Chiropr Man Ther. 2015;23:31

16. Albracht K, Arampatzis A, Baltzopoulos V. Assessment of muscle volume and physiological cross-sectional area of the human triceps surae muscle in vivo. J Biomech. 2008;41:2211-8. https://linkinghub.elsevier.com/retri eve/pii/S0021929008002066. Accessed 22 Apr 2019

17. Stock MS, Mota JA, Hernandez JM, Thompson BJ. Echo intensity and muscle thickness as predictors Of athleticism and isometric strength in middle-school boys. Muscle Nerve. 2017;55:685-92.

18. Nakamura S, Gomez-Tames JD, Gonzalez J, Ojima S, Yamaguchi T, Yu W. Ultrasound imaging and analysis of muscle activity in lower limb. Biosyst Biorobotics. 2013 p. 455-9.

19. Hodges PW, Pengel LHM, Herbert RD, Gandevia SC. Measurement of muscle contraction with ultrasound imaging. Muscle Nerve. 2003;27:682-92.

20. Mickle KJ, Nester CJ, Crofts G, Steele JR. Reliability of ultrasound to measure morphology of the toe flexor muscles. J Foot Ankle Res. 2013;6:12.

21. Jacobs J, Jansen M, Janssen H, Raijmann W, Van Alfen N, Pillen S. Quantitative muscle ultrasound and muscle force in healthy children: A 4-year follow-up study. Muscle Nerve. 2013;47:856-63.

22. Salmi A, Ahovuo J, Tukiainen E, Härmä M, Asko-Seljavaara S. Use of ultrasonography to evaluate muscle thickness and blood flow in free flaps. Microsurgery. 1995;16:601-5.

23. Lin SH, Chuang DCC, Hattori Y, Chen HC. Traumatic major muscle loss in the upper extremity: reconstruction using functioning free muscle transplantation. J Reconstr Microsurg. 2004;20:227-35.

24. Misuri G, Colagrande S, Gorini M, landelli I, Mancini M, Duranti R, et al. In vivo ultrasound assessment of respiratory function of abdominal muscles in normal subjects. Eur Respir J. 1997;10:2861-7. 
25. Maganaris CN, Baltzopoulos V. Predictability of in vivo changes in pennation angle of human tibialis anterior muscle from rest to maximum isometric dorsiflexion. Eur J Appl Physiol Occup Physiol. 1999:79:294-7.

26. Sopher RS, Amis AA, Davies DC, Jeffers JR. The influence of muscle pennation angle and cross-sectional area on contact forces in the ankle joint. J Strain Anal Eng Des. 2017;52:12.

27. Herda TJ, Ryan ED, Kohlmeier M, Trevino MA, Gerstner GR, Roelofs EJ. Examination of muscle morphology and neuromuscular function in normal weight and overfat children aged 7-10 years. Scand J Med Sci Sport. 2018;28:2310-21.

\section{Publisher's Note}

Springer Nature remains neutral with regard to jurisdictional claims in published maps and institutional affiliations.
Ready to submit your research? Choose BMC and benefit from:

- fast convenient online submission

- thorough peer review by experienced researchers in your field

- rapid publication on acceptance

- support for research data, including large and complex data types

- gold Open Access which fosters wider collaboration and increased citations

- maximum visibility for your research: over 100M website views per year

At $B M C$, research is always in progress.

Learn more biomedcentral.com/submissions 